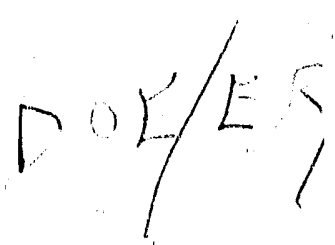

\title{
An Asymptotic Symmetry of the Rapidly Forced Pendulum
}

\author{
by \\ Yi-Hua Chang $t^{\star}$ and Harvey Segur $†$ \\ †Program in Applied Mathematics, University of Colorado, \\ Boulder, CO 80309-0526 \\ *Department of Mathematics, State University of New York \\ Buffalo, NY 14214-3093
}

\section{September 1990}

\section{DISCLAIMER}

This report was prepared as an account of work sponsored by an agency of the United States Government. Neither the United States Government nor any agency thereof, nor any of their Government. Neither the United States Government nor any agency legal liability or responsibility for the accuracy, completeness, or usefulness of any information, apparatus, product, or bility for the accuracy, completeness, or usefulness of any information, appanatus, product, ence herein to any specific commercial product, process, or service by trade name, trademark, ence herein to any specific commercial product, process, or service by trats endorsement, recommanufacturer, or otherwise does not necessarily constitute or imply its endorsement, The views mendation, or favoring by the United States Government or any agency thereof. The views and opinions of authors expressed herein do not 
An Asymptotic Symmetry of the Rapidly Forced Pendulum

b y

Yi-Hua Chang $\dagger^{*}$ and Harvey Segur $\dagger$

†Program in Applied Mathematics, University of Colorado,

Boulder, CO 80309-0526

*Department of Mathematics, State University of New York

Buffalo, NY 14214-3093

\section{Abstract:}

The inhomogeneous differential equation $\left[x^{\prime \prime}+\sin x=\delta \sin \left(t+t_{0}\right) / \varepsilon\right]$ describes the motion of a sinusoidally forced pendulum. The orbits that connect the two saddle points of the unforced $(\delta=0)$ pendulum, $(x=\pi)$ and $(x=-\pi)$, are called separatrices. If $\varepsilon=O(1)$, then one can use Melnikov's method to show that these separatrices can split for weak forcing $(\delta$ « 1$)$, and that the perturbed motion is chaotic. If $\varepsilon$ \& 1 , Melnikov's method fails because the perturbation term is not analytic in $\varepsilon$ at $\varepsilon=0$. In this paper we show that for $\delta$ \& 1 and $\varepsilon$ \& , the solution of the perturbed problem exhibits a symmetry to all orders in an asymptotic expansion. From the asymptotic expansion it follows that the separatrices split by an amount that is at most transcendentally small. This proof differs from that of Holmes, Marsden and Scheurle [1].

Key words: exponentially small, pendulum, asymptotic, Melnikov function, symmetry.

\section{Introduction:}

A frictionless pendulum in a constant gravitational field is perhaps the best-known example of a nonlinear oscillator. The governing equation is

$$
x^{\prime \prime}+\sin x=0
$$

where $x(t)$ is the angle between the arm of the pendulum and a vertical line, and $t$ represents time; the physical parameters of the problem all have been scaled into ( $t$ ). This is a Hamiltonian system with one degree of freedom $\left[H\left(x^{\prime}, x\right)=\left(x^{\prime}\right)^{2} / 2+(1-\cos x)\right]$, and it is integrable; the general solution can be given in terms of elliptic functions. Special solutions of 
interest include a center $[x=0]$, two saddles $[x=\pi, x=-\pi]$, and a heteroclinic orbit (or separatrix),

$$
x_{0}(t)=2 \tan ^{-1}[\sinh (t)]
$$

that connects the two saddles $[x=\pi, x=-\pi]$.

Let us perturb the motion of the pendulum by adding a small periodic forcing, so that (1.1) becomes:

$$
\mathrm{x}^{\prime \prime}+\sin \mathrm{x}=\delta \sin \left(\mathrm{t}+\mathrm{t}_{0}\right) / \varepsilon, \quad 0<\delta \ll 1, \quad \varepsilon>0 .
$$

The system is still Hamiltonian:

$$
H=\left[\left(x^{0}\right)^{2} / 2+1-\cos x\right]+\delta\left[-x \sin \left\{\left(t+t_{0}\right) / \varepsilon\right\}\right]=H_{0}+\delta H_{1},
$$

but the Hamiltonian now depends explicitly on time and sometimes is called a $1^{1 / 2}$ degree of freedom Hamiltonian system.

An interesting question is whether the motion is still integrable, or whether it becomes chaotic under this small perturbation. It is known that if chaos appears, it is likely to appear first in the following way [2]: the heteroclinic orbit $x_{0}(t)$ splits into two orbits; one behaves nicely as $t \rightarrow-\infty$ ( part of the unstable manifold of $x=-\pi$ ), the other behaves nicely as $t \rightarrow+\infty$ ( part of the stable manifold of $x=\pi$ ), but neither orbit is wellbehaved for all $\mathrm{t}(-\infty<\mathrm{t}<+\infty)$. The minimum distance between these two orbits in the 3-dimensional phase space $\left(x, x^{\prime}, t\right)$ is called the "splitting distance". It provides a measurable indicator of chaos:

(i) If this distance vanishes identically in $t_{0}$, then no splitting occurs, there is a heteroclinic orbit in the perturbed problem, and the system remains integrable.

(ii) If the splitting distance has simple zeroes as $t_{0}$ varies, then the stable manifold and unstable manifold intersect transversely in the two dimensional Poincare map, and one can show that the system is chaotic [3,4].

Melnikov [5] showed that if a certain integral (now called "the Melnikov function") has a finite limit as $\delta \rightarrow 0$, then it faithfully approximates this distance in this limit. Thus to demonstrate chaos it is sufficient to show that as $\delta \rightarrow 0$, the Melnikov function approches a finitevalued function of $t_{0}$ with simple zeroes. For (1.3) the Melnikov function is

$$
M\left(\varepsilon, t_{0}\right)=\int_{-\infty}^{\infty} d t\left[\sin \left(t+t_{0}\right) / \varepsilon\right] \cdot\left[x_{0}{ }^{\prime}(t)\right],
$$


and this can be evaluated explicitly:

$$
M\left(\varepsilon, t_{0}\right)=2 \pi[\operatorname{sech}(\pi / 2 \varepsilon)] \cdot\left[\sin \left(t_{0} / \varepsilon\right)\right] .
$$

For any fixed $\varepsilon$, this function is finite-valued except for simple zeroes, so the forced pendulum exhibits chaotic behaviour [3,4].

The situation :s more delicate in the high frequency (or rapidly forced) limit, in which both $\varepsilon \rightarrow 0$ and $\delta \rightarrow 0$. The case of greatest interest occurs when

$$
\delta / \varepsilon \rightarrow 1, \quad \text { as } \varepsilon \rightarrow 0, \delta \rightarrow 0,
$$

because in this case (1.3) represents many Hamiltonian systems in which one has already averaged over oscillations on a fast time-scale [6]. In this case $\mathrm{M}\left(\varepsilon, t_{0}\right)$ still has simple zeroes, but it becomes exponentially small as $\varepsilon \rightarrow 0$, so that Melnikov's theorem no longer applies. Whether $M\left(\varepsilon, t_{0}\right)$ still approximates the actual splitting distance under (1.7) is not known. There has been great interest recently in problems like this one, in which the qualitative character of the solution is controlled by a quantity that is exponentially small [see $7-14$ and the references therein].

\section{Main results:}

The differential equation is:

$$
x^{\prime \prime}+\sin x=\delta \sin \left[\left(t+t_{0}\right) / \varepsilon\right], \quad \delta, \varepsilon \ll 1 .
$$

With $x_{0}(t)$ given by (1.2), set

$$
x\left(t, t_{0}, \varepsilon, \delta\right)=: x_{0}(t)+\delta y\left(t, t_{0}, \varepsilon, \delta\right),
$$

so that $y$ satisfies

where

$$
y^{\prime \prime}+\left(\cos x_{0}\right) y=f(y, t, \delta)+\sin \left[\left(t+t_{0}\right) / \varepsilon\right]
$$

$$
f(y, t, \delta)=-\left\{\left(\sin x_{0}\right)[(\cos \delta y) \cdot 1]+\left(\cos x_{0}\right)[(\sin \delta y)-\delta y]\right\} / \delta .
$$

Let $\mathrm{y}^{+}$denote a solution of (2.3) that is bounded as $t \rightarrow+\infty$, and let $c^{+}:=\left.y^{+}\right|_{t=0}$. Let $y^{-}$denote a solution of $(2.3)$ that is bounded as $t \rightarrow-\infty$, and let $c^{-}:=\left.y^{-1}\right|_{t=0}$. 
Theorem 1: For $t \geq 0$, there is a $\delta_{0}>0$ such that a one-parameter family of solutions, $y^{+}\left(t, t_{0}, \varepsilon, \delta, c^{+}\right)$, of (2.3) exists for $\delta \leq \delta_{0}$, uniformly in $\varepsilon$ for $\varepsilon>0$. Each of these solutions is uniformly bounded for $t \geq 0$. For $t \leq 0$, $y^{*}\left(t, t_{0}, \varepsilon, \delta, c^{*}\right)$ is similarly defined and bounded. (All of the Theorems stated in this section are proved in section 3.)

Now represent $y^{ \pm}\left(t, t_{0}, \varepsilon, \delta, c^{ \pm}\right)$by formal series for $0<\delta \ll 1$ :

$$
\delta y^{ \pm}\left(t, t_{0}, \varepsilon, \delta, c^{ \pm}\right) \sim \sum_{1}^{\infty} \delta^{n} x_{n}^{ \pm}\left(t, t_{0}, \varepsilon, c_{n}^{ \pm}\right)
$$

Substituting (2.4) into (2.3) and equating powers of $\delta$ yields

$$
\left(x_{1}^{ \pm}\right)^{\prime \prime}+\left(\cos x_{0}\right) x_{1}^{ \pm}=\sin \left[\left(t+t_{0}\right) / \varepsilon\right]
$$

and for $n=2,3, \ldots$

$$
\left(x_{n}^{ \pm}\right)^{\prime \prime}+\left(\cos x_{0}\right) x_{n}^{ \pm}=\left(\sin x_{0}\right) \cdot \Phi_{n}^{ \pm}\left(x_{1}, x_{2}, \ldots, x_{n-1}\right)+\left(\cos x_{0}\right) \cdot \Psi_{n}^{ \pm}\left(x_{1}, x_{2} \ldots, x_{n} .\right.
$$
1),

where $\Phi_{n}^{ \pm}$and $\Psi_{n}^{ \pm}$are polynomials in $\left(\mathrm{x}_{1}{ }^{ \pm}, \mathrm{x}_{2}{ }^{ \pm}, \ldots, \mathrm{x}_{\mathrm{n}-1}{ }^{ \pm}\right) ;$see (3.22) below.

For $n=1,2, \ldots$, let $x_{n}{ }^{+}$denote a solution of (2.5) that is bounded as $t \rightarrow+\infty$, and let $c_{n}^{+}:=\left.x_{n}^{+}\right|_{t=0}$. Let $x_{n}^{-}$denote a bounded solution as $t \rightarrow-\infty$, and let $c_{n}{ }^{-}:=\left.x_{n}{ }^{-1}\right|_{t=0}$.

Theorem 2: For every $n \geq 1,(2.5)$ has a one-parameter family of solutions, $x_{n}^{+}\left(t, t_{0}, \varepsilon, c_{n}^{+}\right)$, that are uniformly bounded for $t \geq 0$ and for $\varepsilon>0$. Similarly, every $x_{n}{ }^{-}\left(t, t_{0}, \varepsilon, c_{n}{ }^{-}\right)$is uniformly bounded for $t \leq 0$ and $\varepsilon>0$.

In order for $(2.4)$ to hold at $t=0$, we require that

$$
c^{+} \sim \sum_{1}^{\infty} \delta^{n} c_{n}^{+} \text {and } c^{-} \sim \sum_{1}^{\infty} \delta^{n} c_{n}^{-} \quad \text { as } \delta \rightarrow 0
$$

Theorem 3: Given (2.6), the series in (2.4) are asymptotic to $y^{ \pm}\left(t, t_{0}, \varepsilon, \delta, c^{ \pm}\right)$ as $\delta \rightarrow 0$, uniformly in $\varepsilon$ for $\varepsilon>0$, and uniformly in $t$ for $t \geq 0$, and for $t \leq 0$.

Now we let $\varepsilon \rightarrow 0$ as $\delta \rightarrow 0$. For $n=1,2, \ldots$, we seek expansions of the form: 


$$
x_{n} \pm \sim \sum_{k=1}^{\infty} \varepsilon^{k} \sum_{j=0}^{n}\left\{A_{n k j}(t) \cdot \sin \left[j\left(t+t_{0}\right) / \varepsilon\right]+B_{n k j}(t) \cdot \cos \left[j\left(t+t_{0}\right) / \varepsilon\right]\right\} .
$$

Theorem 4 asserts that such expansions exist for a particular choice of $c_{n}{ }^{ \pm}$, $\mathrm{n}=1,2, \ldots$.

Theorem 4: One can choose $\left\{c_{1}{ }^{ \pm}, c_{2}{ }^{ \pm}, c_{3}{ }^{ \pm}, \ldots\right\}$ so that as $\varepsilon \rightarrow 0$, the series in (2.7) are asymptotic to $x_{n}{ }^{+}\left(t, t_{0}, \varepsilon, c_{n}{ }^{+}\right)$uniformly in $t$ for $t \geq 0$, and to $x_{n}{ }^{\circ}\left(t, t_{0}, \varepsilon, c_{n}{ }^{-}\right)$uniformly for $t \leq 0$. Moreover for each $(n, k, j) A_{n k j}(t)$ and $B_{n k j}(t)$ in (2.7) and all of their derivatives are uniformly bounded in $t$.

This representation leads to our main result, that (2.7) exhibits a symmetry to all orders.

Theorem 5: For every $(n, j, k)$ in (2.7), $A_{n k j}(t)$ is an even function of $t$, and $B_{n k j}(t)$ is an odd function of $t(n, j, k=1,2, \ldots)$. If $n$ is even and $j$ is odd, then $A_{n k j}(t)$ and $B_{n k j}(t)$ vanish for every $k$. If $n$ is odd and $j$ is even, then $A_{n k j}(t)$ and $B_{n k j}(t)$ vanish for every $k$. In particular, if $n$ is odd and $j=0$, then $B_{n k j}(t)$ vanishes.

Finally, the splitting distance $\Delta\left(t_{0}, \varepsilon, \delta\right)$ is defined as follows:

$$
\left[\Delta\left(t_{0}, \varepsilon, \delta\right)\right]^{2}:=\delta^{2}\left[\left.y^{+}\right|_{t=0}-\left.y^{-}\right|_{t=0}\right]^{2}+\delta^{2}\left[\left.\left(y^{+}\right)^{\prime}\right|_{t=0}-\left.\left(y^{-}\right)^{-}\right|_{t=0}\right]^{2} \text {. }
$$

We can require $\left[\left.\mathrm{y}^{+}\right|_{\mathrm{t}=0}=\left.\mathrm{y}^{-}\right|_{\mathrm{t}=0}\right]$, so that (2.8) becomes:

$$
\Delta\left(t_{0}, \varepsilon, \delta\right)=\delta\left|\left[\left.\left(y^{+}\right)^{\prime}\right|_{t=0}-\left.\left(y^{-}\right)^{-}\right|_{t=0}\right]\right|
$$

Let

$$
\Delta_{n}\left(t_{0}, \varepsilon\right):=\left[\left.\left(x_{n}^{+}\right)^{\prime}\right|_{t=0}-\left.\left(x_{n}\right)^{-}\right|_{t=0}\right],
$$

so that

$$
\Delta\left(t_{0}, \varepsilon, \delta\right) \sim \sum_{1} \delta^{n}\left|\Delta_{n}\right|
$$

Theorem 6: As $\varepsilon \rightarrow 0$, for every $n \geq 1, \Delta_{n}$ " $\varepsilon^{k}$ for every positive integer $k$.

This means that the splitting distance is at most transcendentally small in $\varepsilon$ as $\varepsilon \rightarrow 0$. This resuit had previously been obtained by Holmes, Marsden and Scheurle [1], Scheurle [15], and Neishtadt [16], by other means. 


\section{Detailed analysis:}

Lemma 1: The linear operators,

$$
G^{ \pm}\{y(t)\}:=-\alpha_{1}(t) \int_{0}^{t} d s \alpha_{2}(s) y(s)+{ }_{c_{2}}(t) \int_{ \pm \infty}^{t} d s \alpha_{l}(s) y(s)
$$

where $\quad \alpha_{1}(t)=\operatorname{sech} t, \quad \alpha_{2}(t)=(\sinh t+t \operatorname{sech} t) / 2$,

are bounded operators. Specifically, if $y(t)$ is bounded on $0 \leq t<\infty$, so

then

$$
|| y||:=\sup _{0 \leq t<\infty}|y(t)|<\infty,
$$

$$
|| G^{+}\{y\}|| \leq 3|| y||
$$

Similarly, if $y(t)$ is bounded for $t \leq 0$, then

$$
|| G^{-}\{y\}|| \leq 3|| y|| \text {. }
$$

Proof: Suppose $y(t)$ is uniformly bounded for $t \geq 0$. Then $\left\|G^{+}\{y(t)\}\right\| \leq\|y\| \cdot \sup 0 \leq t<\infty\left\{\alpha_{1}(t) \int_{0}^{t} d s \alpha_{2}(s)-\alpha_{2}(t) \int_{\infty}^{t} d s \alpha_{1}(s)\right\}$.

But

$$
\begin{aligned}
& \alpha_{1}(s) \leq 1, \\
& s \bullet(\operatorname{sech} s) \leq 2 s \bullet[\exp (-s)] \leq 2 e^{-1} \leq 1,
\end{aligned}
$$

so

$$
\begin{aligned}
& \alpha_{1}(t) \int_{0}^{t} \alpha_{2}(s) d s \leq(1+\operatorname{sech} t+t \cdot \operatorname{sech} t) / 2 \leq 3 / 2, \\
& \left|\alpha_{2}(t) \int_{\infty}^{t} \alpha_{1}(s) d s\right| \leq-(\sinh t+1) \int_{\infty}^{t} 2 e^{-s} d s \leq 3 / 2,
\end{aligned}
$$

and

$$
\| G^{+}\{y(t)\}|| \leq 3|| y||
$$

The proof of $(3.3 \mathrm{~b})$ is similar.

Q.E.D.

Theorem 1: For $t \geq 0$, there is $a \delta_{0}>0$ such that a one-parameter family of solutions, $y^{+}\left(t, t_{0}, \varepsilon, \delta, c^{+}\right)$, of (2.3) exists for $\delta \leq \delta_{0}$, uniformly in $\varepsilon$ for $\varepsilon>0$. Each of these solutions is uniformly bounded for $t \geq 0$. For $t \leq 0$, $y^{*}\left(t, t_{0}, \varepsilon, \delta, c^{*}\right)$ is similarly defined and bounded. 
Proof: Consider:

$$
y^{ \pm}\left(t, t_{0}, \varepsilon, \delta, c^{ \pm}\right)=: K^{ \pm}\left(y^{ \pm}\right)=c^{ \pm} \alpha_{1}(t)+G^{ \pm}\left\{f\left(y^{ \pm}, t, \delta\right)+\sin \left[\left(t+t_{0}\right) / \varepsilon\right]\right\}
$$

where $c^{ \pm}$are independent of $t$.

First we prove that there is a $\delta_{0}, 0<\delta_{0}<1$, such that for $\delta \leq \delta_{0},(3.4)$ has a unique solution, $\mathrm{y}^{+}\left(\mathrm{t}, \mathrm{t}_{0}, \varepsilon, \delta, \mathrm{c}^{+}\right)$, that is uniformly bounded for $\mathrm{t} \geq 0$ and for $\varepsilon>0$. For $t \leq 0, y^{-}\left(t, t_{0}, \varepsilon, \delta, c^{-}\right)$is similarly defined. We use an iterative method for $\mathrm{y}^{+}$. The proof for $\mathrm{y}^{-}$is similar. For simplicity, we omit the " + " in what follows. The iteration relation is

$$
\mathrm{y}_{\mathrm{n}+1}=\mathrm{K}\left(\mathrm{y}_{\mathrm{n}}\right)
$$

with $\mathrm{y}_{0}=0$.

(i) $y_{1}=K\left(y_{0}\right)=c \alpha_{1}(t)+G\left\{\sin \left[\left(t+t_{0}\right) / \varepsilon\right]\right\}$.

$\| y_{1}||=\sup _{0 \leq t<\infty}\left\{\left|y_{1}(t)\right|\right\}$

$\leq \sup _{0 \leq \mathrm{t}<\infty}\left\{|c| \alpha_{1}(t)+\left|G\left\{\sin \left[\left(t+t_{0}\right) / \varepsilon\right]\right\}\right|\right\} \leq|d|+3=$ : M. (by Lemma 1)

(ii) $y_{2}-y_{1}=G\left\{f\left(y_{1}, s, \delta\right)\right\}=G\left\{-\left(\sin x_{0}\right)\left[\cos \left(\delta y_{1}\right)-1\right]-\left(\cos x_{0}\right)\left[\sin \left(\delta y_{1}\right)-\delta y_{1}\right]\right\} / \delta$.

Because

(1): $\left|\sin x_{0}\right| \leq 1,\left|\cos x_{0}\right| \leq 1$,

(2): $\left|\cos \left(\delta y_{1}\right)-1\right|$

$$
\begin{aligned}
& =\left|-\left(\delta y_{1}\right)^{2} / 2+\left(\delta y_{1}\right)^{4} /(4 !)-\left(\delta y_{1}\right)^{6} /(6 !)+\left(\delta y_{1}\right)^{8} /(8 !)-\ldots\right| \\
& \leq\left(\delta y_{1}\right)^{2}\left|1+\left(\delta y_{1}\right)^{2} / 2+\left(\delta y_{1}\right)^{4} /(4 !)+\left(\delta y_{1}\right)^{6} /(6 !)+\left(\delta y_{1}\right)^{8} /(8 !)+\ldots\right| \\
& \leq\left(\delta y_{1}\right)^{2}\left[\exp \left(\delta^{2} y_{1}^{2}\right)\right]
\end{aligned}
$$

(3): $\left|\sin \left(\delta y_{1}\right)-\delta y_{1}\right|$

$$
\begin{aligned}
& =\left|-\left(\delta y_{1}\right)^{3} /(3 !)+\left(\delta y_{1}\right)^{5} /(5 !)-\left(\delta y_{1}\right)^{7} /(7 !)+\left(\delta y_{1}\right)^{9} /(9 !)-\ldots\right| \\
& \leq\left|\delta y_{1}\right|^{3}\left[\exp \left(\delta^{2} y_{1}^{2}\right)\right],
\end{aligned}
$$

then by Lemma 1, (3.5), (3.6) and (3.7),

||$y_{2}-y_{1}|| \leq 3 \sup _{0 \leq t<\infty}\left\{\left(\delta\left|y_{1}\right|^{2}+\delta^{3}\left|y_{1}\right|^{3}\right)\left[\exp \left(\delta^{2}\left|y_{1}\right|^{2}\right)\right]\right\}$

$\leq 3\left(\delta M^{2}+\delta^{2} M^{3}\right)\left[\exp \left(\delta^{2} M^{2}\right)\right]=3 \delta\left(M^{2}+\delta M^{3}\right)\left[\exp \left(\delta^{2} M^{2}\right)\right]$ 


$$
<3\left(\mathrm{M}^{2}+\mathrm{M}^{3}\right)\left(\exp \mathrm{M}^{2}\right)=: \mathrm{N} \text {. }
$$

(iii) For $k \geq 2$, note that for $\delta<1$,

$$
\begin{aligned}
& |\partial \mathrm{f}(\mathrm{y}, \mathrm{t}, \delta) / \partial \mathrm{y}|=\left|\sin \mathrm{x}_{0}(-\sin \delta \mathrm{y})+\left(\cos \mathrm{x}_{0}\right)(\cos \delta \mathrm{y}-1)\right| \\
& \leq\left(|\delta \mathrm{y}|+|\delta \mathrm{y}|^{2} / 2\right)=\delta\left(|\mathrm{y}|+\delta|\mathrm{y}|^{2} / 2\right) \leq \delta\left(2|\mathrm{y}|+|\mathrm{y}|^{2}+1\right)=\delta(|\mathrm{y}|+1)^{2} \\
& \leq \delta(|| \mathrm{y}||+1)^{2}=\mathrm{L} \text {. }
\end{aligned}
$$

The Lipschitz constant is $\mathrm{L}$, so

$$
|f(y, t, \delta)-f(z, t, \delta)| \leq L|y-z| \text {. }
$$

Therefore

$$
\begin{aligned}
& \left\|y_{k+1}-y_{k}\right\|=\sup _{0 \leq t<\infty}\left\{G\left\{f\left(y_{k}, t, \delta\right)-f\left(y_{k-1}, t, \delta\right)\right\}\right\} \leq 3 \sup _{0 \leq t<\infty}\left\{L\left|y_{k-1} y_{k-1}\right|\right\} \\
& \quad \leq 3 L \bullet\left\|y_{k-y_{k-1}}\right\| \leq(3 L)^{2} \bullet|| y_{k-1-y_{k-2}}\left\|\leq \ldots \leq(3 L)^{k-1} \bullet\right\| y_{2-y_{1}} \| .
\end{aligned}
$$

(iv) $y(t)=\lim _{n \rightarrow \infty} y_{n}(t)=y_{0}(t)+\sum_{n=0}^{\infty}\left[y_{n+1}(t)-y_{n}(t)\right]$, so

$$
\begin{aligned}
& |y(t)| \leq \sum_{n=0}^{\infty}\left|y_{n+1}(t)-y_{n}(t)\right|=\left|y_{1}(t)\right|+\left|y_{2}(t)-y_{1}(t)\right|+\left|y_{3}(t)-y_{2}(t)\right|+\ldots \\
& \leq\left\|y_{1}\right\|+\left\|y_{2}-y_{1}\right\|\left\{1+(3 L)+(3 L)^{2}+\ldots+(3 L)^{n}+\ldots\right\}=\| y_{1}||+|| y_{2}-y_{1}|| /(1-3 L) \\
& \leq M+N /(1-3 L),
\end{aligned}
$$

where $M, N$ were defined in (3.5), (3.8). Now we choose $\delta_{0}$ by requiring

$$
3 L=3 \delta(\|y\|+1)^{2} \leq 0.9 \text {. }
$$

Then from $(3.12)$

$$
\|y\| \leq M+N /(1-0.9)=M+10 N
$$

so from (3.13)

$$
\delta \leq(0.3) /(\mathrm{M}+10 \mathrm{~N}+1)^{2}=: \delta_{0}
$$

To summarize, for $0 \leq \delta \leq \delta_{0}, y(t)$ is defined by (3.4), and it is uniformly bounded by (3.14) for $\mathrm{t} \geq 0$ and $\varepsilon>0$. 
(v) To show that the solution of (3.4) is unique, suppose $y(t)$ and $z(t)$ both solve (3.4) for $t \geq 0$. Then from (3.4), (3.10), and Lemma 1 ,

$$
\|y-z\|=\|G\{f(y, t, \delta)\}-G(f(z, t, \delta)\}\| \leq 3 L\|y-z\| .
$$

But $3 L \leq 0.9$ (i.e., this is a contraction mapping), so $\| y-z||=0$, and the solution is unique.

Thus, for each fixed constant $\left(c^{+}\right),(3.4)$ defines $y^{+}\left(t, t_{0}, \varepsilon, \delta, c^{+}\right)$ uniquely. By differentiating (3.4), one verifies that $y^{+}$is twice-differentiable, and that it satisfies (2.3). This completes the proof of Theorem 1 for $t \geq 0$. The proof for $t \leq 0$ is similar.

QED.

Theorem 2: For every $n \geq 1,(2.5)$ has a one-parameter family of solutions, $x_{n}{ }^{+}\left(t, t_{0}, \varepsilon, c_{n}{ }^{+}\right)$, that are uniformly bounded for $t \geq 0$ and for $\varepsilon>0$. Similarly, every $x_{n}{ }^{-}\left(t, t_{0}, \varepsilon, c_{n}{ }^{-}\right)$is uniformly bounded for $t \leq 0$ and $\varepsilon>0$.

Proof: (i) By formally solving (2.5a) for $t \geq 0$, we obtain

$$
\mathrm{x}_{1}^{+}\left(\mathrm{t}, \mathrm{t}_{0}, \varepsilon, \mathrm{c}_{1}^{+}\right)=\mathrm{K}_{1}^{+}\left\{\left[\sin \left(\mathrm{t}+\mathrm{t}_{0}\right) / \varepsilon\right]\right\}=\mathrm{c}_{1}^{+} \alpha_{1}(\mathrm{t})+\mathrm{G}^{+}\left\{\left[\sin \left(\mathrm{t}+\mathrm{t}_{0}\right) / \varepsilon\right]\right\} .
$$

Using Lemma 1,

$$
|| x_{1}+||=\sup _{0 \leq t<\infty}\left|x_{1}{ }^{+}\right| \leq\left|c_{1}^{+}\right|+3
$$

so for fixed $\mathrm{c}_{1}{ }^{+}, \mathrm{x}_{1}{ }^{+}$is uniformly bounded for $\mathrm{t} \geq 0$ and $\varepsilon>0$. By differentiating (3.16), one shows that $x_{1}{ }^{+}$is twice-differentiable, and that it satisfies (2.5a). Evaluating (3.16) at $t=0$ shows that $\left.x_{1}{ }^{+}\right|_{t=0}=c_{1}{ }^{+}$. For $t \leq 0$, a similar argument applies to $x_{1}^{-}\left(t, t_{0}, \varepsilon, c_{1}^{-}\right)$.

(ii) For $n=2,3, \ldots$, and $t \geq 0$, formally solving (2.5b) yields

$$
\begin{array}{r}
x_{n}^{+}\left(t, t_{0}, \varepsilon, c_{n}^{+}\right)=: K_{n}^{+}\left\{\left(\sin x_{0}\right) \cdot \Phi_{n}^{+}+\left(\cos x_{0}\right) \cdot \Psi_{n}^{+}\right\} \\
=c_{n}^{+} \alpha_{1}(t)+G^{+}\left\{\left(\sin x_{0}\right) \cdot \Phi_{n}^{+}+\left(\cos x_{0}\right) \cdot \Psi_{n}^{+}\right\}
\end{array}
$$

where $\Phi_{\mathrm{n}}^{+}$and $\Psi_{\mathrm{n}}^{+}$are polynomials in $\left(\mathrm{x}_{1}^{+}, \mathrm{x}_{2}{ }^{+}, \ldots, \mathrm{x}_{\mathrm{n}-1}{ }^{+}\right)$. Now we use induction. Suppose that $\left(\mathrm{x}_{1}^{+}, \mathrm{x}_{2}{ }^{+}, \ldots, \mathrm{x}_{\mathrm{n}-1}{ }^{+}\right)$all are uniformly bounded for $t \geq 0$. Then

$$
|| \Phi_{n}^{+}||<\infty, \quad|| \Psi_{n}{ }^{+} \|<\infty
$$


and by Lemma 1 ,

$$
|| x_{n}+|\leq| c_{n}^{+} \mid+3\left(|| \Phi_{n}{ }^{+}||+|| \Psi_{n}^{+}||\right)
$$

so $x_{n}{ }^{+}$is uniformly bounded for $t \geq 0$. Differentiating (3.17) shows that $x_{n}{ }^{+}$ satisfies (2.5b), and evaluating (3.17) at $t=0$ shows that $\left.x_{n}{ }^{+}\right|_{t=0}=c_{n}{ }^{+}$. Similar arguments apply to $x_{n}{ }^{-}\left(t, t_{0}, \varepsilon, c_{n}{ }^{-}\right)$.

Q.ED.

Theorem 3: Given (2.6), the series in (2.4) are asymptotic to $y^{ \pm}\left(t, t_{0}, \varepsilon, \delta, c^{ \pm}\right)$as $\delta \rightarrow 0$, uniformly in $\varepsilon$ for $\varepsilon>0$, and uniformly in $t$ for $t \geq 0$, and for $t \leq 0$.

Proof: We give the proof for $y^{+}\left(t, t_{0}, \varepsilon, \delta, c^{+}\right)$; the proof of $y^{-}\left(t, t_{0}, \varepsilon, \delta, c^{-}\right)$is similar, and we omit it. Also, we omit the "+" in the following proof.

Recall that " $\mathrm{f}(\delta)=\mathrm{O}(\delta)$ " means that there is a fixed $\mathrm{W}<\infty$ and a $\delta_{0}>0$, such that

$$
|f(\delta)| \leq W \delta \text { for } 0<\delta \leq \delta_{0}
$$

(i) We want to prove: $\left(y-x_{1}\right)=O(\delta)$. From (3.4) and (3.16),

$$
\left(y-x_{1}\right)=\left(c-c_{1}\right) \alpha_{1}+G\{f(y, t, \delta)\} .
$$

But by (2.6),

$$
c-c_{1}=O(\delta) \text {, }
$$

and by Lemma $1,(3.6)$ and (3.7),

$$
|| G\{f(y, t, \delta)\}|| \leq 3 \delta \cdot\left(\|y\|^{2}+\delta \mid y \|^{3}\right) \cdot \exp (\delta\|y\|)^{2},
$$

so for $\mathrm{t} \geq 0$ and $\varepsilon>0$,

$$
\left(y-x_{1}\right)=O(\delta)
$$

(ii) We want to prove: $\left\{y-x_{1}-\delta x_{2}\right\} / \delta=O(\delta)$. For any analytic function $F(z)$, let $\left[F\left(\Sigma_{1}{ }^{\infty} \delta^{k} a_{k}\right)\right]_{n}$ denote the $n^{\text {th }}$ truncation of its $\delta$-power series: 


$$
\begin{aligned}
& {\left[F\left(\Sigma_{1}^{\infty} \delta^{k} a_{k}\right)\right]_{n}=\left[\Sigma_{1}^{\infty} \delta^{k} b_{k}\right]_{n}=\Sigma_{1}{ }^{n} \delta^{k} b_{k} .} \\
& \left\{y-x_{1}-\delta x_{2}\right\} / \delta=\left\{c-c_{1}-\delta c_{2}\right\} \alpha_{1} / \delta+G\left\{f(y, t, \delta)-\left(\sin x_{0}\right)\left[\cos \left(\delta x_{1}\right)-1\right]_{2} / \delta\right\} / \delta .
\end{aligned}
$$

By (2.6),

$$
\left\{c-c_{1}-\delta c_{2}\right\} \alpha_{1} / \delta=O(\delta) \text {. }
$$

From (2.3b),

$$
f(y, t, \delta)=\left(\sin x_{0}\right)\left(\delta y^{2} / 2\right)+O\left(\delta^{2}\right)
$$

Also

$$
\left[\cos \left(\delta x_{1}\right)-1\right]_{2} / \delta=\delta x_{1}^{2} / 2 \text {, }
$$

so that

$$
\left\{f(y, t, \delta)-\left(\sin x_{0}\right)\left[\cos \left(\delta x_{1}\right)-1\right]_{2} / \delta\right] / \delta=\left(\sin x_{0}\right)\left(y-x_{1}\right)\left(y+x_{1}\right) / 2+O(\delta)=O(\delta),
$$

by (3.18). Then using Lemma 1 shows that

$$
\left\{y-x_{1}+\delta x_{2}\right\} / \delta=O(\delta) \text {. }
$$

(iii) We want to prove: $\left.\left\{y-x_{1}-\delta x_{2}-\delta^{2} x_{3}\right)\right\} / \delta^{2}=0(\delta)$.

$$
\begin{aligned}
& \left\{y-x_{1}-\delta x_{2}-\delta^{2} x_{3}\right\} / \delta^{2} \\
& =\left(1 / \delta^{2}\right)\left(\left[c-c_{1}-\delta c_{2}-\delta^{2} c_{3}\right] \alpha_{1}-G\left\{\left(\sin x_{0}\right)\left\{(\cos \delta y)-1-\left[\cos \left(\sum_{n=1}^{2} \delta^{n} x_{n}\right)-1\right]_{3}\right\}\right.\right. \\
& \left.\left.+\left(\cos x_{0}\right)\left\{(\sin \delta y)-\delta y-\left[\sin \left(\sum_{n=1}^{2} \delta^{n} x_{n}\right)-\left(\sum_{n=1}^{2} \delta^{n} x_{n}\right)\right]_{3}\right\}\right\} / \delta\right) .
\end{aligned}
$$

But

$$
\begin{aligned}
& (\cos \delta y)-1-\left[\cos \left(\sum_{n=1}^{2} \delta^{n} x_{n}\right)-1\right]_{3}=(\cos \delta y)-\cos \left(\sum_{n=1}^{2} \delta^{n} x_{n}\right)+O\left(\delta^{4}\right) \\
& =2 \sin \left\{\left(\delta y-\sum_{n=1}^{2} \delta^{n} x_{n}\right) / 2\right\} \cdot \sin \left\{\left(\delta y+\sum_{n=1}^{2} \delta^{n} x_{n}\right) / 2\right\}+O\left(\delta^{4}\right) \\
& =O\left(\delta^{3}\right) \cdot O(\delta)+O\left(\delta^{4}\right),
\end{aligned}
$$

and

$$
\begin{aligned}
& (\sin \delta y)-\delta y-\left[\sin \left(\delta x_{1}\right)-\left(\delta x_{1}\right)\right]_{3}=\left(\sin \delta y-\sin \delta x_{1}\right)-\left(\delta y-\delta x_{1}\right)+O\left(\delta^{4}\right) \\
& =2 \sin \left\{\left(\delta y-\delta x_{1}\right) / 2\right\} \cos \left\{\left(\delta y+\delta x_{1}\right) / 2\right\}-\left(\delta y-\delta x_{1}\right)+O\left(\delta^{4}\right) \\
& =2 \sin \left\{\left(\delta y-\delta x_{1}\right) / 2\right\}-\left(\delta y-\delta x_{1}\right)+O\left(\delta^{4}\right)=O\left(\delta^{4}\right)
\end{aligned}
$$

Then the result follows by using (2.6) and Lemma 1 . 
(iv) In the general case, we want to prove:

$$
\left\{y-\left(\sum_{n=1}{ }^{m} \delta^{n-1} x_{n}\right)\right\}=O\left(\delta^{m}\right)
$$

The proof is by induction. Assume that (3.21) holds for $m=1,2, \ldots, k$. Then

$$
\left[y-\left(\sum_{n=1}^{k+1} \delta^{n-1} x_{n}\right)\right] / \delta^{k}
$$

$=\left(1 / \delta^{k}\right)\left(\left[c-\sum_{n=1}^{k+1} \delta^{n-1} c_{n}\right] \alpha_{1}-G\left\{\left(\sin x_{0}\right)\left\{(\cos \delta y)-1-\left[\cos \left(\sum_{n=1}{ }^{k} \delta^{n} x_{n}\right)-1\right]_{k+1}\right\} / \delta\right.\right.$

$$
\left.\left.+\left(\cos x_{0}\right)\left\{(\sin \delta y)-\delta y-\left[\sin \left(\sum_{n=1}^{k-1} \delta^{n} x_{n}\right)-\left(\sum_{n=1}^{k-1} \delta^{n} x_{n}\right)\right]_{k+1}\right\} / \delta\right\}\right) .
$$

But

$$
\begin{aligned}
& (\cos \delta y)-1-\left[\cos \left(\sum_{n=1}{ }^{k} o^{n} x_{n}\right)-1\right]_{k+1}=(\cos \delta y)-\cos \left(\sum_{n=1}^{k} \delta^{n} x_{n}\right)+O\left(\delta^{k+2}\right) \\
& =2 \sin \left(\left(\delta y-\sum_{n=1} \delta^{n} x_{n}\right) / 2\right\} \sin \left\{\left(\delta y+\sum_{n=1}^{k} \delta^{n} x_{n}\right) / 2\right\}+O\left(\delta^{k+2}\right) \\
& =O\left(\delta^{k+1}\right) \cdot O(\delta)+O\left(\delta^{k+2}\right),
\end{aligned}
$$

and

$$
\begin{aligned}
& (\sin \delta y)-\delta y-\left[\sin \left(\sum_{n=1}^{k-1} \delta^{n} x_{n}\right)-\left(\sum_{r=1}^{k-1} \delta^{n} x_{n}\right)\right]_{k+1} \\
& =(\sin \delta \mathrm{y})-\sin \left(\sum_{\mathrm{n}=1}^{\mathrm{k}+1} \delta^{\mathrm{n}} \mathrm{x}_{\mathrm{n}}\right)-\left\{\delta \mathrm{y}-\left(\sum_{\mathrm{n}=1}^{\mathrm{k}+1} \delta^{\mathrm{n}} \mathrm{x}_{\mathrm{n}}\right)\right\}+\mathrm{O}\left(\delta^{\mathrm{k}+2}\right) \\
& =2 \sin \left\{\left(\delta y-\sum_{n=1}^{k-1} \delta^{n} x_{n}\right) / 2\right\} \cos \left\{\left(\delta y+\sum_{n=1}^{k-1} \delta^{n} x_{n}\right) / 2\right\}-\left\{\delta y-\sum_{n=1}^{k-1} \delta^{n} x_{n}\right\}+O\left(\delta^{k+2}\right) \\
& =O\left(\delta^{3 k}\right)+O\left(\delta^{k+2}\right)=O\left(\delta^{k+2}\right) \text {. }
\end{aligned}
$$

In order to prove Theorem 4 first we note that in $(2.5 \mathrm{~b})$,

$$
\begin{aligned}
& \Phi_{2}^{ \pm}\left(x_{1}\right)=\left(x_{1}^{ \pm}\right)^{2} / 2, \quad \Psi_{2}^{ \pm}\left(x_{1}\right)=0, \\
& \Phi_{3}{ }^{ \pm}\left(x_{1}, x_{2}\right)=x_{1}^{ \pm} \bullet x_{2}^{ \pm}, \quad \Psi_{3}^{ \pm}\left(x_{1}, x_{2}\right)=\left(x_{1}^{ \pm}\right)^{3} / 6, \\
& \Phi_{4}^{ \pm}\left(x_{1}, x_{2}, x_{3}\right)=\left(x_{2}^{ \pm}\right)^{2} / 2+x_{1}^{ \pm} \bullet x_{3}{ }^{ \pm}-\left(x_{1}^{ \pm}\right)^{4} /(4 !), \\
& \Psi_{4}^{ \pm}\left(x_{1}, x_{2}, x_{3}\right)=\left(x_{1}^{ \pm}\right)^{2} \bullet\left(x_{2}{ }^{ \pm}\right) / 2 .
\end{aligned}
$$

In general,

$$
\Sigma_{2}^{\infty} \delta^{n} \Phi_{n}^{ \pm}=\Sigma_{1}^{\infty}(-1)^{k}\left[\left(\Sigma \delta^{n} x_{n}^{ \pm}\right)^{2 k}\right] /(2 k) !,
$$




$$
\Sigma_{3}^{\infty} \delta^{n} \Phi_{n}^{ \pm}=\Sigma_{2}^{\infty}(-1)^{k-1}\left[\left(\Sigma \delta^{n} x_{n}^{ \pm}\right)^{2 k-1}\right] /(2 \mathrm{k}-1) !
$$

so

$$
\begin{aligned}
& \Phi_{n}^{ \pm}\left(x_{1}, x_{2}, \ldots x_{n-1}\right)=\Sigma_{\left(b_{1}, b_{2}, \ldots, b_{n-1}\right)} C_{\left(b_{1}, b_{2}, \ldots, b_{n-1}\right)}\left(x_{1}^{ \pm}\right)^{b_{1}}\left(x_{2}^{ \pm}\right)^{b_{2}} \ldots\left(x_{n-1}^{ \pm}\right)^{b_{n-1}}, \\
& b_{1}+2 b_{2}+\ldots+(n-1) b_{n-1}=n,\left(b_{1}+b_{2}+\ldots+b_{n-1}\right): \text { even } \\
& \left.\Psi_{n}^{ \pm}\left(x_{1}, x_{2}, \ldots x_{n-1}\right)=\Sigma_{\left(b_{1}, b_{2}, \ldots, b_{n-1}\right)} D_{\left(b_{1}, b_{2}, \ldots, b_{n-1}\right)}\left(x_{1}^{ \pm}\right)^{b_{1}}\left(x_{2}{ }^{ \pm}\right)^{b_{2}} \ldots\left(x_{n-1}{ }^{ \pm}\right)^{b_{n-1}},\right\}
\end{aligned}
$$

where $b_{i} \in(0,1,2, \ldots, n)$, and $\left\{C_{\left(b_{1}, b_{2}, \ldots, b_{n-1}\right)}\right.$ and $\left.D_{\left(b_{1}, b_{2}, \ldots, b_{n-1}\right)}\right\}$ are pure constants.

Lemma 2: Suppose $f(t), g(t)$, and all of their derivatives are uniformly bounded on $-\infty<t<\infty$. With $\alpha_{1}(t), \alpha_{2}(t)$ defined in (3.1), let

and

$$
\begin{aligned}
I_{l}^{ \pm}=d_{l}^{ \pm} \alpha_{l}(t)-\alpha_{l}(t) & \int_{0}^{t} \alpha_{2}(s) f(s) \sin \left[k\left(s+t_{0}\right) / \varepsilon\right] d s \\
& +\alpha_{2}(t) \int_{ \pm \infty}^{t} \alpha_{l}(s) f(s) \sin \left[k\left(s+t_{0}\right) / \varepsilon\right] d s
\end{aligned}
$$

$$
\begin{aligned}
I_{2}{ }^{ \pm}=d_{2}{ }^{ \pm} \alpha_{l}(t)-\alpha_{1}(t) & \int_{0}^{t} \alpha_{2}(s) g(s) \cos \left[k\left(s+t_{0}\right) / \varepsilon\right] d s \\
& +\alpha_{2}(t) \int_{ \pm \infty}^{t} \alpha_{l}(s) g(s) \cos \left[k\left(s+t_{0}\right) / \varepsilon\right] d s,
\end{aligned}
$$

where $\left\{d_{1}^{ \pm}, d_{2}^{ \pm}\right\}$are independent of $t$, and $k$ is a positive integer.

Then there is a choice of $\left\{d_{1}^{ \pm}(\varepsilon), d_{2}^{ \pm}(\varepsilon)\right\}$ so that

$$
I_{j}=R_{j}(t, \varepsilon) \sin k \theta+S_{j}(t, \varepsilon) \cos k \theta
$$

where $j=1$ or $2, \theta=\left(t+t_{0}\right) / \varepsilon$, and $R_{j}(t, \varepsilon)$ and $S_{j}(t, \varepsilon)$ are given by asymptotic power-series, as follows, For $N \geq 1$,

$$
\begin{aligned}
& R_{l}(t, \varepsilon)=\Sigma_{n=0} N(-1)^{n}(\varepsilon / k)^{2 n+2}\left\{-\alpha_{1}\left[\alpha_{2} f\right]^{(2 n+1)}+\alpha_{2}\left[\alpha_{l} f\right]^{(2 n+1)}\right\}+o\left(\varepsilon^{N}\right), \\
& S_{l}(t, \varepsilon)=\Sigma_{n=1} N(-1)^{n-1}(\varepsilon / k)^{2 n+1}\left\{-\alpha_{1}\left[\alpha_{2} f\right]^{(2 n)}+\alpha_{2}\left[\alpha_{l} f\right]^{(2 n)}\right\}+o\left(\varepsilon^{N}\right), \\
& R_{2}(t, \varepsilon)=\Sigma_{n=1} N(-1)^{n+1}(\varepsilon l k)^{2 n+1}\left\{-\alpha_{1}\left[\alpha_{2} g\right]^{(2 n)}+\alpha_{2}\left[\alpha_{1} g\right]^{(2 n)}\right\}+o\left(\varepsilon^{N}\right),
\end{aligned}
$$




$$
S_{2}(t, \varepsilon)=\Sigma_{n=0} N(-1)^{n}(\varepsilon / k)^{2 n+2}\left\{-\alpha_{1}\left[\alpha_{2} g\right]^{(2 n+1)}+\alpha_{2}\left[\alpha_{1} g\right]^{(2 n+1)}\right\}+o\left(\varepsilon^{N}\right),
$$

where $\left[J^{(n)}:=\left(d^{n} / d t^{n}\right)[\right.$ J for $n=1,2, \ldots$. These series need not converge as $N \rightarrow \infty$, but for each series, every truncation at some finite $N$ is uniformly bounded in $t$, along with all of its t-derivatives.

Proof: (i) If $f(t)$ and all of its derivatives are uniformly bounded, then for $n \geq 1, \alpha_{2}(t)\left[\alpha_{1}(t) f(t)\right]^{(n)}$ and $\alpha_{1}(t)\left[\alpha_{2}(t) f(t)\right]^{(n)}$ are uniformly bounded, and $\left[\alpha_{1}(t) f(t)\right]^{(n)} \rightarrow 0$ as $t \rightarrow \pm \infty$.

(ii) By repeatedly integrating $\mathrm{I}_{1}^{+}$by parts, and choosing $\mathrm{d}_{1}^{+}(\varepsilon)$ to cancel the sequence of boundary contributions at $t=0$, we obtain

$$
\begin{aligned}
\mathrm{I}_{1}^{+}= & (\varepsilon / \mathbf{k})\left\{-\alpha_{1}\left[\alpha_{2} \mathrm{f}\right]+\alpha_{2}\left[\alpha_{1} \mathrm{f}\right]\right\}(-\operatorname{cosk} \theta) \\
& +(\varepsilon / k)^{2}\left\{-\alpha_{1}\left[\alpha_{2} f\right]^{(1)}+\alpha_{2}\left[\alpha_{1} f\right]^{(1)}\right\}(\operatorname{sink} \theta) \\
& +(\varepsilon / k)^{3}\left\{-\alpha_{1}\left[\alpha_{2} f\right]^{(2)}+\alpha_{2}\left[\alpha_{1} f^{(2)}\right\}(\operatorname{cosk} \theta)\right. \\
& +(\varepsilon / k)^{4}\left\{-\alpha_{1}\left[\alpha_{2} f\right]^{(3)}+\alpha_{2}\left[\alpha_{1} f\right]^{(3)}\right\}(-\operatorname{sink} \theta) \\
& +(\varepsilon / k)^{5}\left\{-\alpha_{1}\left[\alpha_{2} f\right]^{(4)}+\alpha_{2}\left[\alpha_{1} f\right]^{(4)}\right\}(-\operatorname{cosk} \theta) \\
& +\ldots \\
& +(-1)^{n-1}(\varepsilon / k)^{2 n+1}\left\{-\alpha_{1}\left[\alpha_{2} f\right]^{(2 n)}+\alpha_{2}\left[\alpha_{1} f\right]^{(2 n)}\right\}(\operatorname{cosk} \theta) \\
& +(-1)^{n}(\varepsilon / k)^{2 n+2}\left\{-\alpha_{1}\left[\alpha_{2} f\right]^{(2 n+1)}+\alpha_{2}\left[\alpha_{1} f\right]^{(2 n+1)}\right\}(\operatorname{sink} \theta) \\
& +(-1)^{n+1}(\varepsilon / k)^{2 n+2}\left\{-\alpha_{1}(t) \int_{0}^{t}\left[\alpha_{2}(s) f(s)\right]^{(2 n+2)} \sin \left\{k\left(s+t_{0}\right) / \varepsilon\right\} d s\right. \\
& \left.+\alpha_{2}(t) \int_{+\infty}^{t}\left[\alpha_{1}(s) f(s)\right]^{(2 n+2)} \sin \left\{k\left(s+t_{0}\right) / \varepsilon\right\} d s\right\}
\end{aligned}
$$

(iii) Now (3.24) follows for $\mathrm{I}_{1}^{+}$, if we can bound the two integral remainders uniformly in $t$ and $\varepsilon$. The first integral is

$$
\left|\alpha_{1}(t) \int_{0}^{t}\left[\alpha_{2}(s) f(s)\right]^{(2 n+2)} \sin \left(k\left(s+t_{0}\right) / \varepsilon\right\} d s\right|
$$




$$
\begin{aligned}
& \leq \alpha_{1}(t) \int_{0}^{t}\left|\left[\alpha_{2}(s) f(s)\right]^{(2 n+2)}\right| d s \\
& \leq \alpha_{1}(t) \int_{0}^{t}\left|\sum_{j=0}^{2 n+2} H_{j}\left[\alpha_{2}(s)\right]^{(j)}[f(s)]^{(2 n+2-j)}\right| d s \\
& \leq \sum_{\mathrm{j}=0}^{2 n+2} \mathrm{H}_{\mathrm{j}} \sup _{0 \leq s<\infty}||[\mathrm{f}(\mathrm{s})]^{(2 n+2-j)}\left|! \alpha_{1}(\mathrm{t}) \int_{0}^{\mathrm{t}}\right|\left[\alpha_{2}(\mathrm{~s})\right]^{(\mathrm{j})} \mid \mathrm{ds},
\end{aligned}
$$

where $\mathrm{H}_{\mathrm{j}}$ is constant $(\mathrm{j}=0,1, \ldots, 2 \mathrm{n}+2)$. For $\mathrm{j}=0,1, \ldots, 2 \mathrm{n}+2$ one can show that $\alpha_{1}(\mathrm{t}) \int_{0}^{\mathrm{t}}\left|\left[\alpha_{2}(\mathrm{~s})\right]^{(j)}\right| \mathrm{ds} \leq \mathrm{M}_{\mathrm{j}}<\infty$,

where $M_{j}$ is constant, so the first integral is bounded. Similarly, one can show that the second integral is bounded. This establishes (3.24) for $\mathrm{I}_{1}{ }^{+}$. The proofs for $\mathrm{I}_{1}^{-}$and $\mathrm{I}_{2}^{ \pm}$are similar, and we omit them.

Finally, the uniform boundedness of any $t$-derivative of any truncation of $R_{j}(t, \varepsilon)$ or $S_{j}(t, \varepsilon)$ follows because $\left\{\alpha_{1}(t),\left[\alpha_{1}(t) \alpha_{2}(t)\right], f(t)\right.$ and $g(t)\}$ all are uniformly bounded, along with all of their derivatives. Q.E.D.

Theorem 4: One can choose $\left\{c_{1}^{ \pm}, c_{2}^{ \pm}, c_{3}{ }^{ \pm}, \ldots\right\}$ so that as $\varepsilon \rightarrow 0$, the series in (2.7) are asymptotic to $x_{n}{ }^{+}\left(t, t_{0}, \varepsilon, c_{n}{ }^{+}\right)$uniformly in $t$ for $t \geq 0$, and io $x_{n}{ }^{-}\left(t, t_{0}, \varepsilon, c_{n}{ }^{-}\right)$uniformly for $t \leq 0$. Moreover for each $(n, k, j) A_{n k j}(t)$ and $B_{n k j}(t)$ in (2.7) and all of their derivatives are uniformly bounded in $t$.

Proof: We use induction on $n$.

(i) For $n=1, x_{1}{ }^{+}$is defined by (3.16). This representation is in the form (3.23a), with $f=1, k=1$ and $c_{1}{ }^{+}=d_{1}{ }^{+}$. Then the result follows from Lemma 2 .

(ii) Suppose for $n=1,2, \ldots, m-1$, each $x_{n}{ }^{+}$has a representation in the form of (2.7) with $A_{n k j}(t), B_{n k j}(t)$, and all of their derivatives uniformly bounded in $t$, for $t \geq 0$, and for every $(n, j, k)$. Then for $n=m$, it follows from (2.7) and (3.22) that $\Phi_{n}^{+}$and $\Psi_{n}^{+}$each can be represented in the form

$\Phi_{n}^{+}=\sum_{k=1}{ }^{k} \varepsilon^{k} \sum_{j=0}{ }^{n}\left\{C_{n k j}(t) \cdot \sin \left[j\left(t+t_{0}\right) / \varepsilon\right]+D_{n k j}(t) \cdot \cos \left[j\left(t+t_{0}\right) / \varepsilon\right]\right\}+o\left(\varepsilon^{k}\right)$.

Moreover, every $C_{n k j}(t)$ and $D_{n k j}(t)$ in (3.25) is uniformly bounded in $t$, as are all of their $t$-derivatives. Moreover, the remainder is $o\left(\varepsilon^{K}\right)$ uniformly in $t$ for $\mathrm{t} \geq 0$.

(iii) Each term with $\mathrm{j}>0$ in the sum in (3.25), when substituted into (3.17), leads to an integral of the form (3.23). By (ii) and Lemma 2, the 
contribution to $x_{n}{ }^{+}$from each of these terms has the form (3.24), and is consistent with (2.7).

(iv) From Lemma 1, every term with $j=0$ in (3.25) contributes to $x_{n}{ }^{+}$a term of the form $\varepsilon^{k} F_{k}(t)$ for some $k \geq 2$, where $F_{k}(t)$ is uniformly bounded for $t \geq 0$. It is straight-forward to show that the derivatives of $F_{k}(t)$ are uniformly bounded as well.

(v) Finally, using Lemma 1 again, one shows that the contribution to $x_{n}{ }^{+}$ from the $o\left(\varepsilon^{\mathrm{K}}\right)$ remainder in (3.25) is $o\left(\varepsilon^{\mathrm{K}}\right)$ as well. Combining (iii), (iv), (v) establishes Theorem 4 for $x_{n}{ }^{+}$. The argument for $x_{n}{ }^{-}$is similar. QED.

In order to prove Theorem 5 , it is convenient to introduce some extra notation. Let $\theta=\left(t+t_{0}\right) / \varepsilon$, and let (2c) denote any function of the form

$$
\left\{D_{0}+D_{1} \cos 2 \theta+D_{2} \cos 4 \theta+\ldots+D_{n} \cos 2 n \theta\right\}
$$

for some $n$, and for some set of constants $\left(D_{0}, \ldots, D_{n}\right\}$. We define four such families of functions:

$$
\begin{aligned}
& (2 c)=\left\{D_{0}+D_{1} \cos 2 \theta+D_{2} \cos 4 \theta+\ldots+D_{n} \cos 2 n \theta\right\} \\
& (2 s)=\left\{D_{1} \sin 2 \theta+D_{2} \sin 4 \theta+\ldots+D_{n} \sin 2 n \theta\right\} \\
& (1 c)=\left\{D_{1} \cos \theta+D_{2} \cos 3 \theta+\ldots+D_{n} \cos (2 n-1) \theta\right\} \\
& (1 s)=\left\{D_{1} \sin \theta+D_{2} \sin 3 \theta+\ldots+D_{n} \sin (2 n-1) \theta\right\}
\end{aligned}
$$

Then one can easily verify the following formulae:
$(2 s)(2 s)=(2 c)$,
(1s) $(1 s)=(2 c)$,
(2c) $(2 c)=(2 c)$,
(1c) $(1 \mathrm{c})=(2 \mathrm{c})$,
(2c) $(2 s)=(2 s)$,
(1s) $(1 c)=(2 s)$,
(1s) $(2 s)=(1 s)$
(1s) $(2 \mathrm{c})=(1 \mathrm{~s})$,
(1c) $(2 c)=(1 c)$,
(1c) $(2 s)=(1 s)$.

More generally, we may replace the constants $\left\{D_{i}\right\}$ in (3.26) by functions of $t$ that are either even or odd, and we define $f_{0}(2 s)=\left\{f_{1}(t)(\sin 2 \theta)+f_{2}(t)(\sin 4 \theta)+\ldots+f_{n}(t)(\sin 2 n \theta) \mid\right.$ if all $f_{i}(t)$ are odd $\}$ 
$f_{e}(2 s)=\left\{f_{1}(t)(\sin 2 \theta)+f_{2}(t)(\sin 4 \theta)+\ldots+f_{n}(t)(\sin 2 n \theta) \mid\right.$ if all $f_{i}(t)$ are even $\}$,

$g_{0}(2 c)=\left\{g_{0}(t)+g_{1}(t)(\cos 2 \theta)+g_{2}(t)(\cos 4 \theta)+\ldots+g_{n}(t)(\cos 2 n \theta) \mid\right.$ if all $g_{i}(t)$ are odd $\}$,

$g_{e}(2 c)=\left\{g_{0}(t)+g_{1}(t)(\cos 2 \theta)+g_{2}(t)(\cos 4 \theta)+\ldots+g_{n}(t)(\cos 2 n \theta) \mid\right.$ if all $g_{i}(t)$ are even $\}$,

$f_{0}(1 s)=\left\{f_{1}(t)(\sin \theta)+f_{2}(t)(\sin 3 \theta)+\ldots+f_{n}(t)[\sin 2(n-1) \theta] \mid\right.$ if all $f_{i}(t)$ are odd $\}$,

$f_{e}(1 s)=\left\{f_{1}(t)(\sin \theta)+f_{2}(t)(\sin 3 \theta)+\ldots+f_{n}(t)[\sin 2(n-1) \theta] \mid\right.$ if all $f_{i}(t)$ are even $\}$,

$g_{0}(1 c)=\left\{g_{1}(t)(\cos \theta)+g_{2}(t)(\cos 3 \theta)+\ldots+g_{n}(t)[\cos 2(n-1) \theta] \mid\right.$ if all $g_{i}(t)$ are odd $\}$,

$g_{e}(1 c)=\left\{g_{1}(t)(\cos \theta)+g_{2}(t)(\cos 3 \theta)+\ldots+g_{n}(t)[\cos 2(n-1) \theta] \mid\right.$ if all $g_{i}(t)$ are even $\}$

Finally, define

$$
\begin{aligned}
& \alpha=f_{o}(2 s)+g_{e}(2 c), \\
& \beta=f_{e}(2 s)+g_{o}(2 c), \\
& \gamma=f_{e}(1 s)+g_{o}(1 c), \\
& \zeta=f_{o}(1 s)+g_{e}(1 c) .
\end{aligned}
$$

Then one can easily verify the following formulae:

$$
\begin{aligned}
& \alpha^{2}=\beta^{2}=\gamma^{2}=\zeta^{2}=\alpha, \\
& \alpha \bullet \beta=\beta \text {, } \\
& \alpha \bullet \gamma=\gamma, \\
& \alpha \cdot \zeta=\zeta \text {, } \\
& \zeta \bullet \gamma=\beta . \\
& \beta \bullet \zeta=\gamma, \\
& \beta \circ \gamma=\zeta \text {, }
\end{aligned}
$$

Now we use this notation to prove some Lemmas that lead up to Theorem 5 . For Lemmas 3, 4, 5, 6, we assume that for $m=1,2, \ldots$,

and $\left\{\begin{array}{l}x_{2 m}=f_{e}(2 s)+g_{o}(2 c)=\beta, \\ x_{2 m-1}=f_{e}(1 s)+g_{o}(1 c)=\gamma\end{array}\right.$

\section{Lemma 3: Given (3.29), then}

$$
\left(x_{n}\right)^{k}= \begin{cases}\alpha, & \text { if } n k: \text { even, } k: \text { even, } \\ \beta, & \text { if } n k: \text { even, } k: \text { odd, } \\ \gamma_{1} & \text { if } n k: \text { odd, }\end{cases}
$$


where $n, k$ are integers.

Proof: There are three cases.

Case I: nk: even, k: even. We want to prove: $\left(x_{n}\right)^{k}=\alpha$.

There are two possibilities: (a) $\mathrm{n}$ : even; and (b) $\mathrm{n}$ : odd,

(a) k: even, n: even.

We use induction in $k$. If $k=2$, then from (3.28) and (3.29), $\left(x_{n}\right)^{2}=\beta \bullet \beta=\alpha$.

Suppose for $k=2 i,\left(x_{n}\right)^{2 i}=\alpha$.

Then for $k=2 i+2,\left(x_{n}\right)^{2 i+2}=\left(x_{n}\right)^{2 i}\left(x_{n}\right)^{2}=\alpha \cdot \alpha=\alpha$.

(b) k: even, n: odd.

We use induction in $k$. $k=2$, then

$\left(x_{n}\right)^{2}=\gamma \gamma=\alpha$.

Suppose for $k=2 i,\left(x_{n}\right)^{2 i}=\alpha$.

Then for $k=2 i+2,\left(x_{n}\right)^{2 i+2}=\left(x_{n}\right)^{2 i}\left(x_{n}\right)^{2}=\alpha \bullet \alpha=\alpha$.

Case II : nk: even, k: odd, $n$ : even. We want to prove: $\left(x_{n}\right)^{k}=\beta$.

We use induction in $k$. If $k=1$, then

$\left(x_{n}\right)^{1}=\beta$.

Suppose for $k=2 i-1,\left(x_{n}\right)^{2 i-1}=\beta$.

Then for $k=2 i+1,\left(x_{n}\right)^{2 i+1}=\left(x_{n}\right)^{2 i-1}\left(x_{n}\right)^{2}=\beta \bullet(\beta \bullet \beta)=\beta \bullet \alpha=\beta$.

Case III : nk: odd, k: odd, n: odd. We want to prove: $\left(x_{n}\right)^{k}=\gamma$.

We use induction in $k$. If $k=1$, then

$\left(x_{\mathrm{n}}\right)^{1}=\gamma$.

Suppose for $k=2 i-1,\left(x_{n}\right)^{2 i-1}=\gamma$.

Then for $k=2 i+1,\left(x_{n}\right)^{2 i+1}=\left(x_{n}\right)^{2 i-1}\left(x_{n}\right)^{2}=\gamma \bullet(\gamma \bullet \gamma)=\gamma \bullet \alpha=\gamma$. Q.E.D. 
Lemma 4: Given (3.29), then

$$
I:=\left(x_{n}\right)^{k}\left(x_{m}\right)^{j}= \begin{cases}\alpha, & \text { if } n k+m j: \text { even, } k+j: \text { even, } \\ \beta, & \text { if } n k+m j: \text { even, } k+j: \text { odd, } \\ \zeta, & \text { if } n k+m j: \text { odd, } k+j: \text { even, } \\ \gamma, & \text { if } n k+m j: \text { odd }, k+j: \text { odd, }\end{cases}
$$

where $n, m, k, j$ are integers.

Proof: We use Lemma 3 repeatedly. There are four cases.

Case I: $n k+m j$ : even, $k+j$ : even. We want to prove: $I=\alpha$.

There are three possibilities:

(a) nk: even, mj: even, $\mathrm{k}$ : even, j: even, $\mathrm{I}=\alpha \cdot \alpha=\alpha$,

(b) $n k$ : even, mj: even, $k$ : odd, $j$ : odd, $I=\beta \bullet \beta=\alpha$,

(c) nk: odd, mj: odd, $\mathrm{k}$ : odd, j: odd, I $=\gamma \gamma \gamma=\alpha$.

Case II: $n k+m j$ : even, $k+j$ : odd. We want to prove $I=\beta$.

There are two possibilities:

(a) nk: even, mj: even, $k$ : odd, j: even, $I=\beta \bullet \alpha=\beta$,

(b) nk: even, mj: even, $k$ : even, $j$ : odd, $I=\alpha \bullet \beta=\beta$.

Case III: $n k+m j$ : odd, $k+j$ : even. We want to prove: $I=\zeta$.

There are two possibilities:

(a) nk: even, mj: odd, $\mathrm{k}$ : odd, j: odd, $\mathrm{I}=\beta \circ \gamma=\zeta$,

(b) nk: odd, mj: even: $\mathrm{k}$ : odd, j: odd, $I=\gamma \circ \beta=\zeta$.

Case IV: $n k+m j:$ ord, $k+j$ : odd. We want to prove: $I=\gamma$.

There are two possibilities:

(a) nk: even, mj: odd, $\mathrm{k}$ : even, $\mathrm{j}$ : odd, $\mathrm{I}=\alpha \cdot \gamma=\gamma$,

(b) nk: odd, mj: even, k: odd, j: even, $\mathrm{I}=\gamma \bullet \alpha=\gamma$.

Lemma 5; Given (3.2.9), then

$$
I=\left(x_{n}\right)^{k}\left(x_{m}\right)^{j}\left(x_{l}\right)^{i}= \begin{cases}\alpha, & \text { if } n k+m j+l i: \text { even, } k+j+i: \text { even, } \\ \beta, & \text { if } n k+m j+l i: \text { even, } k+j+i: \text { odd }, \\ \zeta, & \text { if } n k+m j+l i: \text { odd, } k+j+i: \text { even, } \\ \gamma, & \text { if } n k+m j+l i: \text { odd, } k+j+i: \text { odd, }\end{cases}
$$


where $n, m, l, i, j, k$ are integers.

Proof: We use Lemmas 3 and 4 repeatedly. There are four cases. Case I: $n k+m j+l i$ : even, $k+j+i$ : even. We want to prove: $I=\alpha$. There are three possibilities:

(a) $n k+m j$ : even, li: even, $k+j$ : even, $\mathrm{i}$ : even, $\mathrm{I}=\alpha \bullet \alpha=\alpha$,

(b) $n k+m j$ : e ven, li: even, $k+j$ : odd, $i$ : odd, $I=\beta \bullet \beta=\alpha$,

(c) nk+mj: odd, li: odd, $\mathrm{k}+\mathrm{j}=$ odd, i: odd, $\mathrm{I}=\gamma \cdot \gamma=\alpha$.

Case II: $n k+m j+l i:$ even, $k+j+i$ : odd. We want to prove: $I=\beta$. There are three possibilities:

(a) $n k+m j$ : even, li: even, $k+j$ : even, $i$ : odd, $I=\alpha \cdot \beta=\beta$,

(b) $n k+m j$ : even, li: even, $k+j$ : odd, i: even, $I=\beta \bullet \alpha=\beta$,

(c) $n k+m j:$ odd, li: odd, $k+j$ : even, $i:$ odd, $I=\zeta \bullet \gamma=\beta$.

Case III: $n k+m j+l i:$ odd, $k+j+i$ : even. We want to prove: $I=\zeta$.

There are three possibilities:

(a) $n k+m j$ : even, li: odd, $k+j$ : odd, i: odd, $I=\beta \bullet \gamma=\zeta$,

(b) $n k+m j=:$ dd, li: even, $k+j$ : even, i: even, $l=\zeta \bullet \alpha=\zeta$,

(c) nk+mj: odd, li: even, $k+j$ : odd, i: odd, $I=\gamma \bullet \beta=\zeta$.

Case IV: $n k+m j+l i$ : odd, $k+j+i$ : odd. We want to prove: $I=\gamma$.

There are three possibilities:

(a) nk+mj: even, li: odd, $k+j$ : even, i: odd, $I=\alpha \circ \gamma=\gamma$,

(b) $n k+m j$ : odd, li: even, $k+j$ : even, i: odd, $I=\zeta \bullet \beta=\gamma$,

(c) $n k+m j$ : odd, li: even, $k+j$ : odd, i: even, $I=\gamma \alpha=\gamma$.

Q.E.D.

Lemma 6: Given (3.29), then for $n=1,2, \ldots$, $I:=\left(x_{a_{1}}\right)^{b_{1}}\left(x_{a_{2}}\right)^{b_{2}} \ldots\left(x_{a_{n}}\right)^{b_{n}}=\left\{\begin{array}{l}\alpha, \text { if } a_{1} b_{1}+a_{2} b_{2}+\ldots+a_{n} b_{n}: \text { even, } b_{1}+b_{2}+\ldots+b_{n}: \text { even, } \\ \beta, \text { if } a_{1} b_{1}+a_{2} b_{2}+\ldots+a_{n} b_{n}: \text { even, } b_{1}+b_{2}+\ldots+b_{n}: \text { odd } \\ \zeta, \text { if } a_{1} b_{1}+a_{2} b_{2}+\ldots+a_{n} b_{n}: \text { odd, } b_{1}+b_{2}+\ldots+b_{n}: \text { even } \\ \gamma, \text { if } a_{1} b_{1}+a_{2} b_{2}+\ldots+a_{n} b_{n}: \text { odd, } b_{1}+b_{2}+\ldots+b_{n}: \text { odd, }\end{array}\right.$ where $a_{i}, b_{i}(i=1,2, \ldots, n)$ are integers. 
Proof: We use induction on $n$. If $n=1$, this is Lemma 3 . If $n=2$, this is Lemma 4 , and if $n=3$, this is Lemma 5. Suppose for $n=k$ :

$$
I=\left(x_{a_{1}}\right)^{b_{1}}\left(x_{a_{2}}\right)^{b_{2}} \ldots\left(x_{a_{k}}\right)^{b_{k}}= \begin{cases}\alpha, & \text { if } a_{1} b_{1}+a_{2} b_{2}+\ldots+a_{k} b_{k}: \text { even, } b_{1}+b_{2}+\ldots+b_{k}: \text { ever }, \\ \beta, & \text { if } a_{1} b_{1}+a_{2} b_{2}+\ldots+a_{k} b_{k}: \text { even, } b_{1}+b_{2}+\ldots+b_{k}: \text { odd } \\ \zeta, & \text { if } a_{1} b_{1}+a_{2} b_{2}+\ldots+a_{k} b_{k}: \text { odd, } b_{1}+b_{2}+\ldots+b_{k}: \text { even, } \\ \gamma, & \text { if } a_{1} b_{1}+a_{2} b_{2}+\ldots+a_{k} b_{k}: \text { odd, } b_{1}+b_{2}+\ldots+b_{k}: \text { odd }\end{cases}
$$

Then we must show that for $n=k+1$,

$$
I=\left(x_{a_{1}}\right)^{b_{1}} \ldots\left(x_{a_{k+1}}\right)^{b_{k+1}}=\left\{\begin{array}{l}
\alpha, \text { if } a_{1} b_{1}+a_{2} b_{2}+\ldots+a_{k+1} b_{k+1}: \text { even, } b_{1}+b_{2}+\ldots+b_{k+1}: \text { even } \\
\beta, \text { if } a_{1} b_{1}+a_{2} b_{2}+\ldots+a_{k+1} b_{k+1}, \text { even, } b_{1}+b_{2}+\ldots+b_{k+1}: \text { odd } \\
\zeta, \text { if } a_{1} b_{1}+a_{2} b_{2}+\ldots+a_{k+1} b_{k+1}=: \text { odd }, b_{1}+b_{2}+\ldots+b_{k+1}: \text { even } \\
\gamma, \text { if } a_{1} b_{1}+a_{2} b_{2}+\ldots+a_{k+1} b_{k+1}=: \text { odd, } b_{1}+b_{2}+\ldots+b_{k+1}: \text { odd }
\end{array}\right.
$$

The method of proof for $n=k+1$ is the same as that used in proof of Lemma 5. QED.

Lemma 7: Suppose $f(t)$ is uniformly bounded, and $f(-t)=-f(t)$. Consider the operators, $G^{+}\{f\}$ and $G^{-}\{f\}$, from (3.1):

$$
G^{ \pm}\{f\}(t)=-\alpha_{1}(t) \int_{0}^{t} \alpha_{2}(s) \cdot f(s) d s+\alpha_{2}(t) \int_{ \pm \infty}^{t} \alpha_{l}(s) \cdot f(s) d s
$$

Then: (i) $G^{+}\{f\}=G^{-}\{f\}$, and

$$
\text { (ii) } G^{-}\{f\}(-t)=-G^{-}\{f\}(t) \text {. }
$$

Proof: (i) From (3.1),

$$
G^{+}[f]-G^{-}[f]=-\alpha_{2}(t) \int_{-\infty}^{\infty} \alpha_{1}(s) \cdot f(s) d s .
$$

But $\alpha_{1}(s)$ is even, $f(s)$ is odd, so the integral vanishes.

(ii) Using the facts that $\alpha_{2}$ and $f$ are odd, while $\alpha_{1}$ is even, we have

$$
G^{-}\{f\}(-t)=-\alpha_{1}(-t) \int_{0}^{-t} \alpha_{2}(s) \cdot f(s) d s+\alpha_{2}(-t) \int_{-\infty}^{-t} \alpha_{1}(s) \cdot f(s) d s
$$




$$
\begin{aligned}
& =\alpha_{1}(\mathrm{t}) \int_{0}^{\mathrm{t}} \alpha_{2}(-\mathrm{s}) \cdot \mathrm{f}(-\mathrm{s}) \mathrm{ds}+\alpha_{2}(\mathrm{t}) \int_{\infty}^{\mathrm{t}} \alpha_{1}(-\mathrm{s}) \cdot \mathrm{f}(-\mathrm{s}) \mathrm{d} s \\
& =\alpha_{1}(\mathrm{t}) \int_{0}^{\mathrm{t}} \alpha_{2}(\mathrm{~s}) \cdot \mathrm{f}(\mathrm{s}) \mathrm{ds}-\alpha_{2}(\mathrm{t}) \int_{\infty}^{\mathrm{t}} \alpha_{1}(\mathrm{~s}) \cdot \mathrm{f}(\mathrm{s}) \mathrm{ds}=-\mathrm{G}^{+}\{\mathrm{f}\}(\mathrm{t})=-\mathrm{G}^{-}\{\mathrm{f}\}(\mathrm{t}) .
\end{aligned}
$$

Q.ED.

Now we return to the integrals discussed in Lemma 2.

Lemma 8: Suppose $f(t), g(t)$ and all of their derivatives are uniformly bounded on $-\infty<t<\infty$. Define $I_{1}^{ \pm}$and $I_{2}^{ \pm}$by (3.23), so that as $\varepsilon \rightarrow 0$ each has $a$ representation of the form (3.24). Then as $\varepsilon \rightarrow 0$,

(i) if $f(t)=f(-t)$, then $I_{l} \sim R_{l e}(t, \varepsilon) \sin k \theta+S_{l o}(t, \varepsilon) \cos k \theta$,

(ii) if $f(t)=-f(-t)$, then $I_{I} \sim R_{I o}(t, \varepsilon) \sin k \theta+S_{l e}(t, \varepsilon) \cos k \theta$,

(iii) if $g(t)=g(-t)$, then $I_{2} \sim R_{2 o}(t, \varepsilon) \sin k \theta+S_{2 e}(t, \varepsilon) \cos k \theta$,

(iv) if $g(t)=-g(-t)$, then $I_{2} \sim R_{2 e}(t, \varepsilon) \sin k \theta+S_{2 o}(t, \varepsilon) \cos k \theta$.

Proof: (i) From Lemma 2, for any $\mathrm{N} \geq 1$, as $\varepsilon \rightarrow 0$,

$$
\begin{aligned}
I_{1}= & R_{1}(t, \varepsilon) \sin k \theta+S_{1}(t, \varepsilon) \cos k \theta \\
& =(\sin k \theta)\left\{\sum_{n=0} N(-1)^{n}(\varepsilon / k)^{2 n+2}\left\{-\alpha_{1}\left[\alpha_{2} f\right]^{(2 n+1)}+\alpha_{2}\left[\alpha_{1} f\right]^{(2 n+1)}\right\}\right\} \\
& +(\cos k \theta)\left\{\Sigma_{n=1} N(-1)^{n-1}(\varepsilon / k)^{2 n+1}\left\{-\alpha_{1}\left[\alpha_{2} f\right]^{(2 n)}+\alpha_{2}\left[\alpha_{1} f\right]^{(2 n)}\right\}\right\}+o\left(\varepsilon^{N}\right)
\end{aligned}
$$

Recall from (3.1) that $\alpha_{1}(t)$ is even, and $\alpha_{2}(t)$ is odd. Therefore if $f(t)$ is even, then $\alpha_{1}(t) f(t)$ is even, $\alpha_{2}(t) f(t)$ is odd, $\left[\alpha_{1}(t) f(t)\right]^{(2 n)}$ is even, $\left[\alpha_{2}(t) f(t)\right]^{(2 n)}$ is odd, $\alpha_{2}(t)\left[\alpha_{1}(t) f(t)\right]^{(2 n)}$ is odd, and $\alpha_{1}(t)\left[\alpha_{2}(t) f(t)\right]^{(2 n)}$ is odd. Therefore every finite truncation of $S_{1}(t, \varepsilon)$ is odd in $t$. Similarly, one shows that every finite truncation of $R_{1}(t, \varepsilon)$ is even. This establishes (i). Similar proofs lead to (ii), (iii), and (iv).

Q.ED.

Combining the results of Lemmas 2, 7, and 8 leads to Lemma 9.

Lemma 9: For any $n \geq 2$ in (3.22), suppose that as $\varepsilon \rightarrow 0$, the asymptotic representation of $\left\{\sin x_{0} \Psi_{n}^{ \pm}+\cos x_{0} \Phi_{n}^{ \pm}\right\}$takes one of the forms $[\alpha, \beta, \gamma, \zeta]$ defined in (3.27). Choose $c_{n}^{ \pm}(\varepsilon)$ as in Theorem 4. Then the asymptotic representation of $x_{n}^{ \pm}$has the same form $[\alpha, \beta, \gamma, \zeta]$. In other words, $\alpha, \beta, \gamma, \zeta$, are each asymptotically invariant under any of the operators $\left\{K_{n}^{ \pm}\right\}$, for $n \geq 2$. 
Lemma 10: For $n=1,2, \ldots$, as $\varepsilon \rightarrow 0$,

$$
x_{n} \sim \begin{cases}f_{e}(2 s)+g_{o}(2 c)=\beta, & n: \text { even, } \\ f_{e}(1 s)+g_{o}(1 c)=\gamma, & n: \text { odd },\end{cases}
$$

For $n=2,3, \ldots$, as $\varepsilon \rightarrow 0$,

$$
\left[\left(\sin x_{0}\right) \cdot \Phi_{n}{ }^{ \pm}+\left(\cos x_{0}\right) \cdot \Psi_{n}^{ \pm}\right] \sim \begin{cases}f_{e}(2 s)+g_{0}(2 c)=\beta, & n: \text { even, } \\ f_{e}(1 s)+g_{o}(1 c)=\gamma, & n: \text { odd } .\end{cases}
$$

Proof: We use induction in $2 \mathrm{n}$.

(i) For $n=1$, by (3.16), (3.27), Lemmas 2 and 8 , as $\varepsilon \rightarrow 0$,

$$
x_{1}^{ \pm}=K_{1}^{ \pm}\left[\sin \left(t+t_{0}\right) / \varepsilon\right] \sim f_{0}(1 s)+g_{0}(1 c)=\gamma .
$$

(ii) For $n=2$, by Lemma $3,(3.27)$ and the fact that [ $\left.\sin x_{0}(t)\right]$ is odd, as $\varepsilon \rightarrow 0$,

$$
\left[\left(\sin x_{0}\right) \cdot \Phi_{2}{ }^{ \pm}+\left(\cos x_{0}\right) \cdot \Psi_{2}^{ \pm}\right]=\left[\sin x_{0}(t)\right]\left(x_{1}{ }^{ \pm}\right)^{2} / 2 \sim\left[\sin x_{0}(t)\right] \cdot \alpha=\beta .
$$

Then from (3.17) and Lemma 9, as $\varepsilon \rightarrow 0$,

$$
\mathrm{x}_{2}^{ \pm}=\mathrm{K}_{2}^{ \pm}\left[\left(\sin \mathrm{x}_{0}\right) \cdot \Phi_{2}^{ \pm}+\left(\cos \mathrm{x}_{0}\right) \cdot \Psi_{2}^{ \pm}\right] \sim \mathrm{K}_{2}^{ \pm}(\beta) \sim \beta .
$$

(iii) Suppose that as $\varepsilon \rightarrow 0,(3.30)$ holds for $n=1,2, \ldots, 2 k-1,2 k$. Then for $n=2 k+1$, as $\varepsilon \rightarrow 0$, by (3.22) and Lemma 6 ,

$$
\left(\sin x_{0}\right) \cdot \Phi_{2 k+1} \pm\left(\cos x_{0}\right) \cdot \Psi_{2 k+1}^{ \pm} \sim\left(\sin x_{0}\right) \cdot \zeta+\left(\cos x_{0}\right) \cdot \gamma=\gamma
$$

and by Lemma 9 ,

$$
x_{2 k+1}^{ \pm}=K_{2 k+1}^{ \pm}\left[\left(\sin x_{0}\right) \cdot \Phi_{2 k+1}{ }^{ \pm}+\left(\cos x_{0}\right) \cdot \Psi_{2 k+1}^{ \pm}\right] \sim K_{2 k+1}^{ \pm}(\gamma) \sim \gamma .
$$

Similarly for $n=2 k+2$, as $\varepsilon \rightarrow 0$, by (3.22) and Lemma 6 ,

$$
\left(\sin x_{0}\right) \cdot \Phi_{2 k+2}^{ \pm}+\left(\cos x_{0}\right) \cdot \Psi_{2 k+2}^{ \pm} \sim\left(\sin x_{0}\right) \cdot \alpha+\left(\cos x_{0}\right) \cdot \beta=\beta,
$$

and by Lemma 9 ,

$$
x_{2 k+2}^{ \pm}\left(t, t_{0}, \varepsilon\right)=K_{2 k+2}^{ \pm}\left[\left(\sin x_{0}\right) \cdot \Phi_{2 k-1}{ }^{ \pm}+\left(\cos x_{0}\right) \cdot \Psi_{2 k-1}{ }^{ \pm}\right] \sim K_{2 k+2}^{ \pm}(\beta) \sim \beta .
$$


Now Theorem 5 follows directly from Lemma 10.

Theorem 5: For every $(n, j, k)$ in (2.7), $A_{n k j}(t)$ is an even function of $t$, and $B_{n k j}(t)$ is an odd function of $t(n, j, k=1,2, \ldots)$. If $n$ is even and $j$ is odd, then $A_{n k j}(t)$ and $B_{n k j}(t)$ vanish for every $k$. If $n$ is odd and $j$ is even, then $A_{n k j}(t)$ and $B_{n k j}(t)$ vanish for every $k$. In particular, if $n$ is odd and $j=0$, then $B_{n k j}(t)$ vanishes.

A consequence of this asymptotic structure is that the splitting distance, defined in the Introduction, is smaller than any power of $\varepsilon$ as $\varepsilon \rightarrow 0$ and $\delta \rightarrow 0$, because every $\Delta_{n}$ in $(2.11)$ has this property.

Theorem 6: As $\varepsilon \rightarrow 0$, for every $n \geq 1, \Delta_{n}=o\left(\varepsilon^{k}\right)$ for every positive integer $k$.

Proof: (i) For every $n \geq 1$, the $t$-derivatives of $x_{n}{ }^{ \pm}$are obtained by differentiating integral representations like these in (3.16) and (3.17). Substituting these into (2.10) yields, for $n=1$,

$$
\begin{gathered}
\Delta_{1}\left(t_{0}, \varepsilon\right)=\left.\left(x_{1}^{+}\right)^{\cdot}\right|_{t=0}-\left.\left(x_{1}^{-}\right)^{\cdot}\right|_{t=0}=-\int_{-\infty}^{\infty} \alpha_{1}(s) \cdot \sin \left[\left(s+t_{0}\right) / \varepsilon\right] d s \\
=-M\left(t_{0}, \varepsilon\right)=-2 \pi\left[\sin \left(t_{0} / \varepsilon\right)\right] \cdot \operatorname{sech}(\pi / 2 \varepsilon) .
\end{gathered}
$$

This is the usual Melnikor function, and it becomes exponentially small as $\varepsilon \rightarrow 0$.

(ii) For $n \geq 2$, one obtains

$$
\begin{aligned}
\Delta_{n}\left(t_{0}, \varepsilon\right) & =\left.\left(x_{n}^{+}\right)^{\prime}\right|_{t=0}-\left.\left(x_{n}^{-}\right)^{\prime}\right|_{t=0} \\
& =-\int_{-\infty}^{\infty} \alpha_{1}(s) \cdot\left[\left(\sin x_{0}\right) \cdot \Phi_{n}+\left(\cos x_{0}\right) \cdot \Psi_{n}\right] d s,
\end{aligned}
$$

where

$$
\left[\left(\sin x_{0}\right) \cdot \Phi_{n}+\left(\cos x_{0}\right) \cdot \Psi_{n}\right]= \begin{cases}{\left[\left(\sin x_{0}\right) \cdot \Phi_{n}{ }^{+}+\left(\cos x_{0}\right) \cdot \Psi_{n}{ }^{+}\right],} & s \geq 0 \\ {\left[\left(\sin x_{0}\right) \cdot \Phi_{n}{ }^{-}+\left(\cos x_{0}\right) \cdot \Psi_{n}{ }^{-}\right]} & s \leq 0\end{cases}
$$

Making use of (3.31), we obtain for $n \geq 2$ and any positive integer $k$,

$$
\Delta_{n}=\int_{-\infty}^{\infty} \alpha_{1}(s) \beta(\text { or } \gamma) \mathrm{ds}+\int_{-\infty}^{\infty} \alpha_{1}(s) o\left(\varepsilon^{k}\right) \mathrm{d} s
$$




$$
=\int_{-\infty}^{\infty} \alpha_{1}(s) \beta(\text { or } \gamma) \mathrm{d} s+o\left(\varepsilon^{k}\right)
$$

(iii) By (2.7), (3.25), (3.26) and (3.27), one knows that for finite $k$ and $n$, $\beta(s)=f_{e}(2 s)+g_{0}(2 c)=\Sigma_{j=1}{ }^{k} \varepsilon^{j} \sum_{m=0}{ }^{n / 2}\left[f_{j m e}(s)(\sin 2 m \theta)+g_{j m o}(s)(\cos 2 m \theta)\right]$,

and

$$
\begin{array}{rl}
\gamma(s)=f_{e}(1 s)+g_{0}(1 c)=\Sigma_{j=1} & k{ }^{j} \Sigma_{m=1}^{(n+1) / 2}\left\{f_{j m e}(s)[\sin (2 m-1) \theta]\right. \\
& \left.+g_{j m o}(s)(\cos (2 m-1) \theta]\right\} .
\end{array}
$$

In (3.34), moreover, every coefficient $\left\{f_{j m e}(s)\right.$ or $\left.g_{j m o}(s)\right\}$ is uniformly bounded in $t$, along with all of its derivatives.

(iv) Every integral obtained from $\left\{\int_{-\infty}^{\infty} \alpha_{1}(s) \gamma d s\right\}$ contains a rapidly oscillatory function $[\sin (j \theta)$ or $\cos (j \theta)]$, so the contribution from each of these integrals can be shown to be $o\left(\varepsilon^{k}\right)$, by repeatedly integrating by parts and using the vanishing of $\alpha_{1}(s)$ and its derivatives as $s \rightarrow \pm \infty$.

(v) Similarly, the integrals obtained from $\left\{\int_{-\infty}^{\infty} \alpha_{1}(s) \beta d s\right\}$, except for those corresponding to $m=0$, are $o\left(\varepsilon^{k}\right)$ by the same reasoning.

(vi) Every integral corresponding to $m=0$ in $\left\{\int_{-\infty}^{\infty} \alpha_{1}(s) \beta d s\right\}$ vanishes, because $\mathrm{g}_{\mathrm{jmo}}(\mathrm{s})$ is odd and bounded, while $\alpha_{1}(\mathrm{~s})$ is even and integrable. (vii) Combining these results, one obtains that for $n \geq 2, \Delta_{n}=o\left(\varepsilon^{k}\right)$ for every positive integer $\mathbf{k}$.

Q.E.D.

Explicit calculations of $\Delta_{n}\left(t_{0}, \varepsilon\right)$, order by order, suggest that as $\varepsilon \rightarrow 0$,

$$
\Delta_{\mathrm{n}}=\mathrm{O}(\exp [-\pi / 2 \varepsilon])
$$

This was shown explicitly for $n=1$ in (3.32). We believe this to be true for every $n$, but we have not found a general proof.

Acknowledgement: This work was supported in part by the Dep't of Energy Grant \#DE-SG02-86ER25021. 
References:

[1] P.Holmes, J.Marsden and J.Scheurle, Contemporary Mathematics 81 (1988) 213.

[2].M.V.Berry, AIP Conference Proceedings 46 "Topics in nonlinear dynamics", ed: Siebe Jorna. (1978) 16.

[3] J.Guckenheimer and P.J.Holmes, Nonlinear oscillations, dynamical systems, and the bifurcation of vector fields (Applied Mathematical Sciences, Springer-Verlag, N.Y., 1983).

[4] S.Wiggins, Global bifurcations and chaos (Applied Mathematical Sciences, Springer-Verlag, N.Y., 1988).

[5] V.K.Melnikov, Trans. Moscow. Math. Soc. 12 (1963) 1.

[6] J.A.Sanders, Celestial Mechanics 28 (1982) 171.

[7] R.E.Meyer, J. Appl. Math. and Physics (ZAMP) 27 (1976) 181.

[8] H.Segur and M.Kruskal, Phys. Rev. Lett. 58 (1987) 747.

[9] M.Kruskal and H.Segur, "Asymptotics beyond all orders in a model of crystal growth", Stud. App. Math., to appear.

[10] C.J.Amick and J.B.McLeod, Arch. Rational Mech. Anal. 109 (1990) 139.

[11] J.Hammersley and G.Mazzarino, IMA J. Appl. Math. 42 (1989) 43.

[12] H.Segur, "Asymptotics beyond all orders-a survey", to appear in Proc. of Chaos in Australia, ed. by G.Brown.

[13] P.Pelce, ed, Dynamics of curved fronts (Academic Press Inc., Boston, 1988).

[14] D.A.Kessler, J.Koplik and H.Levine, Adv. Phys. 37 (1988) 255.

[15] J.Scheurle, Contemporary Mathematics 97 (1989) 411.

[16] A.Neishtadt, P.M.M. USSR 48 (1984) 133. 




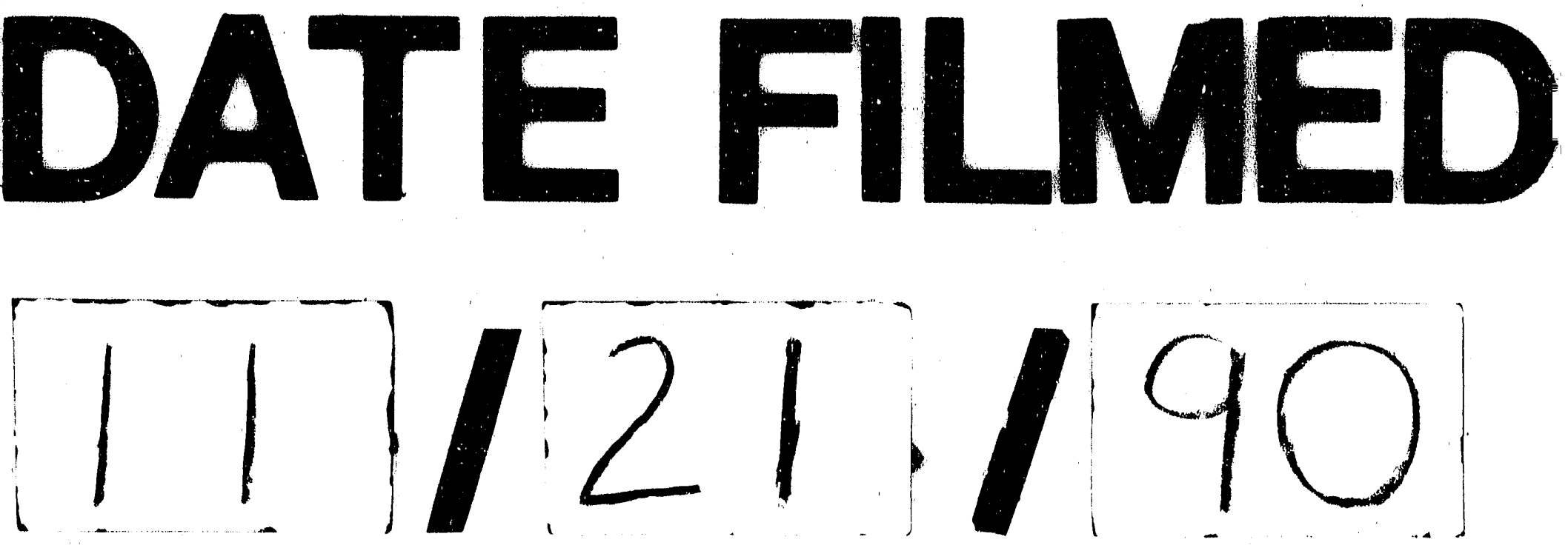
